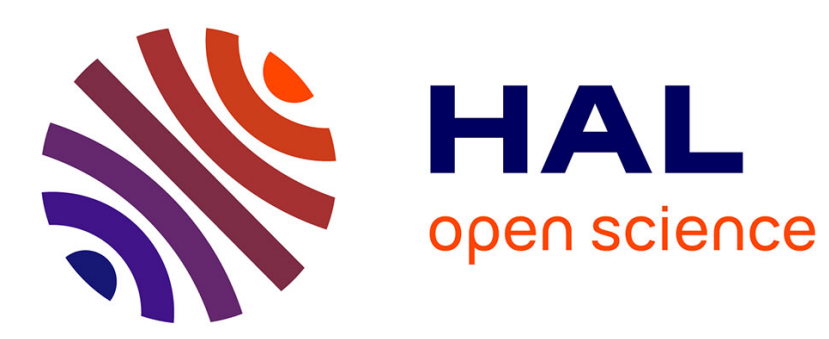

\title{
Penning and associative ionisation of highly excited rubidium atoms
}

\author{
Michel Chéret, Luc Barbier, Werner Lindinger, Robert Deloche
}

\section{To cite this version:}

Michel Chéret, Luc Barbier, Werner Lindinger, Robert Deloche. Penning and associative ionisation of highly excited rubidium atoms. Journal of Physics B: Atomic and Molecular Physics, 1982, 15, pp.3463 - 3477. 10.1088/0022-3700/15/19/015 . cea-01498986

\section{HAL Id: cea-01498986 https://hal-cea.archives-ouvertes.fr/cea-01498986}

Submitted on 30 Mar 2017

HAL is a multi-disciplinary open access archive for the deposit and dissemination of scientific research documents, whether they are published or not. The documents may come from teaching and research institutions in France or abroad, or from public or private research centers.
L'archive ouverte pluridisciplinaire HAL, est destinée au dépôt et à la diffusion de documents scientifiques de niveau recherche, publiés ou non, émanant des établissements d'enseignement et de recherche français ou étrangers, des laboratoires publics ou privés. 
Penning and associative ionisation of highly excited rubidium atoms

This content has been downloaded from IOPscience. Please scroll down to see the full text.

1982 J. Phys. B: At. Mol. Phys. 153463

(http://iopscience.iop.org/0022-3700/15/19/015)

View the table of contents for this issue, or go to the journal homepage for more

Download details:

IP Address: 132.166.19.136

This content was downloaded on 30/03/2017 at 16:31

Please note that terms and conditions apply.

You may also be interested in:

Experimental study of Penning and Hornbeck-Molnar ionisation of rubidium atoms excited in a high $\mathrm{s}$ or d level (5dnl11s)

L Barbier and $\mathrm{M}$ Cheret

Energy pooling process in rubidium vapour

L Barbier and $\mathrm{M}$ Cheret

Crossed-beam measurements of absolute rate coefficients in associative ionisation collisions between $\mathrm{Na}^{*}(\mathrm{np})$ and $\mathrm{Na}(3 \mathrm{~s})$ for $5 \mathrm{n} 15$

$\mathrm{J}$ Boulmer, R Bonanno and J Weiner

Theoretical interpretation of Penning and associative ionisation in collisions between two excited rubidium atoms

L Barbier, A Pesnelle and M Cheret

Hornbeck-Molnar ionisation in a K-Rb mixture

M T Djerad, M Cheret and F Gounand

Collisional ionisation of highly excited rubidium $\mathrm{S}$ state

M Cheret, A Spielfiedel, R Durand et al.

Exchange and direct mechanisms in heteronuclear alkali Penning ionisation

M T Djerad, M Cheret and F Gounand

Super-elastic effects in laser-induced ionisation of Na vapour

B Carre, F Roussel, P Breger et al. 


\title{
Penning and associative ionisation of highly excited rubidium atoms
}

\author{
M Chéret, L Barbier, W Lindinger and R Deloche \\ Service de Physique des Atomes et des Surfaces, Centre d'Etudes Nucleaires de Saclay, \\ 91191 Gif-sur-Yvette Cedex, France
}

Received 5 April 1982, in final form 21 June 1982

\begin{abstract}
Highly excited and resonant states are created in a rubidium vapour in order to study the collisional ionisation with the ground state and between these different excited species. A mass analysis allows us to separate the atomic and molecular channels. Penning ionisation with a resonant state is shown to have a great efficiency (rate coefficient greater than $\left.10^{-8} \mathrm{~cm}^{3} \mathrm{~s}^{-1}\right)$ for all the highly exited levels under study $(6 \mathrm{~d}, 8 \mathrm{~s}, 7 \mathrm{~d})$. The associative ionisation rate coefficient between these excited atoms and a ground-state atom lies in the expected range, $10^{-10}$ to $5 \times 10^{-10} \mathrm{~cm}^{3} \mathrm{~s}^{-1}$ and seems to be independent of $l$ and $j$ values.
\end{abstract}

\section{Introduction}

Mass analysis of ions produced in a $\mathrm{Rb}$ vapour where atomic excited states are created by a two-step photoexcitation technique allows a convenient means for the identification of the main reaction channels producing these ions. In a previous paper (Chéret et al 1981) we have presented a measurement of the rate coefficient for associative ionisation when $\mathrm{Rb}(9 \mathrm{~s})$ excited atoms collide with ground-state atoms. In a second paper (Chéret et al 1982) it has been shown that atomic ions are produced with a large rate coefficient $\left(\sim 10^{-8} \mathrm{~cm}^{3} \mathrm{~s}^{-1}\right)$ by collision between two short-lifetime excited atoms, one in the 8 s state the other in a 5 p state.

The results are interesting for ionised gases or high-density excited vapour studies (Roussel et al 1980, 1981, Lucatorto and Mcllrath 1976, Allegrini et al 1981, Le Gouët et al 1982).

To complement this work a systematic study involving other excited states has been performed. Two important collisional processes have been pointed out.

Penning ionisation (rate coefficient $k_{1}(n l)$ )

$$
\mathrm{Rb}(n l)+\mathrm{Rb}(5 \mathrm{p}) \rightarrow \mathrm{Rb}^{+}+\mathrm{e}^{-}+\mathrm{Rb}(5 \mathrm{~s}) .
$$

Associative ionisation (rate coefficient $k_{2}(n l)$ )

$$
\mathrm{Rb}(n l)+\mathrm{Rb}(5 \mathrm{~s}) \rightarrow \mathrm{Rb}_{2}^{+}+\mathrm{e}^{-} .
$$

The results obtained for the rate coefficients for these two mechanisms are presented for the $6 \mathrm{~d}, 8 \mathrm{~s}$ and $7 \mathrm{~d}$ levels.

† Institut für Experimentalphysik Abteilung, Karl Schönherr strasse 3, A 6020, Innsbruck, Austria. 
In our experimental conditions it is shown that photoionisation processes (cross section $\left.\sigma\left(n l, \lambda_{i}\right)\right)$ play an important role

$$
\mathrm{Rb}(n l)+h \nu \rightarrow \mathrm{Rb}^{+}+\mathrm{e}^{-} .
$$

The experimental technique is presented and the results are discussed. It is shown that other mechanisms producing atomic or molecular ions are negligible under our experimental conditions.

\section{Experimental set-up}

The reaction chamber is a flat stainless steel cylinder $25 \mathrm{~cm}$ in diameter and $8 \mathrm{~cm}$ high (figure 1). Four diameters are used for photoionisation and optical measurements. A mass spectrometer set on the vertical axis of the cell in a separated differentially pumped chamber performs ion analysis. The ions are extracted from the reaction chamber by weak electric fields $\left(\mathrm{a}\right.$ few $\mathrm{V} \mathrm{cm}^{-1}$ ) through a small thin hole (with diameter $1 \mathrm{~mm}$ ). The temperature of the whole apparatus is $450 \mathrm{~K}$ and a heated reservoir filled with rubidium metal is used to maintain a constant pressure of alkali vapour in the range from $10^{-5}$ to $10^{-3}$ Torr. Under these conditions the $\mathrm{Rb}$ concentration lies in the range $2 \times 10^{11}$ to $2 \times 10^{13} \mathrm{~cm}^{-3}$.

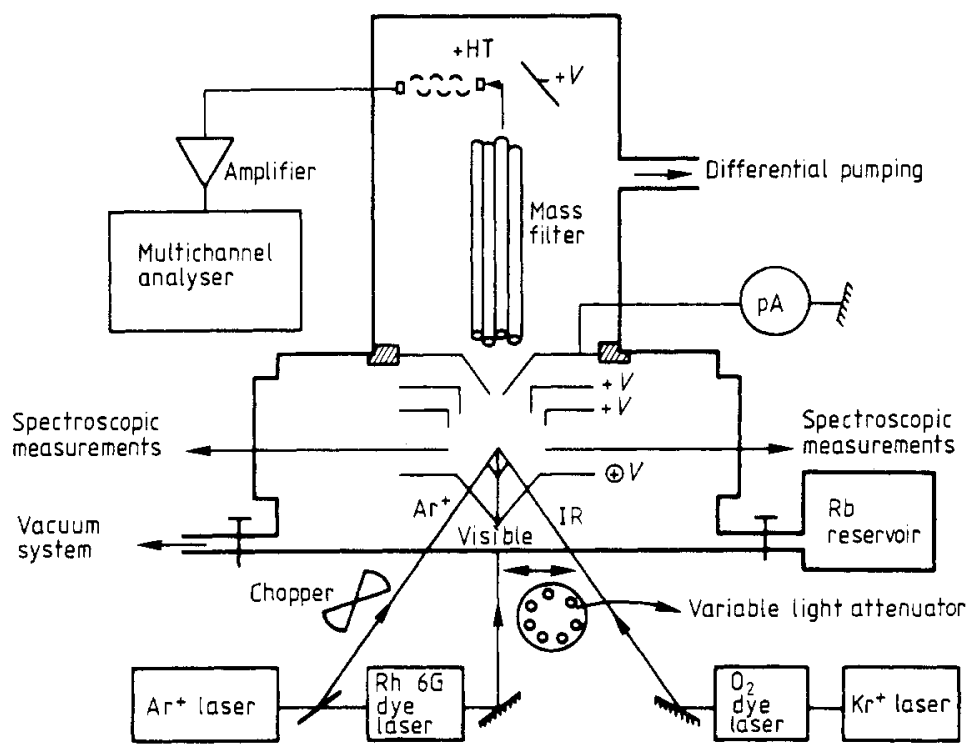

Figure 1. Scheme of the experimental device.

The first $\mathrm{CW}$ infrared dye laser beam $(25 \mathrm{GHz}$ width, laser power in the centre of the cell $P_{1}$, beam section $S_{1}$, wavelength $\lambda_{1}$ ) excites $\mathrm{Rb}$ atoms to one of the first resonant doublets of $\mathrm{Rb}\left(5 \mathrm{p}{ }^{2} \mathrm{P}_{3 / 2}\right)$ at $\lambda_{1}=780.2 \mathrm{~nm}$ or $\mathrm{Rb}\left(5 \mathrm{p}^{2} \mathrm{P}_{1 / 2}\right)$ at $\lambda_{1}=795.0 \mathrm{~nm}$. A second multimode $\mathrm{CW}$ dye laser beam $\left(40 \mathrm{GHz}, P_{2}, S_{2}, \lambda_{2}\right)$ creates $\mathrm{s}$ or $\mathrm{d}$ atomic excited states in the centre of the cell. The overlapping volume of the two laser beams is estimated to be $V=1.0 \times 10^{-2} \mathrm{~cm}^{-3}$. 
According to reactions (1) and (2) these excited states produce atomic and molecular ions. According to reaction (3) photoionisation by the two dye laser beams produces atomic ions. The $\mathrm{Rb}^{+}$and $\mathrm{Rb}_{2}^{+}$ion currents are determined, relative to one another, by the mass spectrometer. The total ion current, which is the sum of these two partial currents, is measured in absolute value on a polarised plate inside the cell. The transmission through our system is assumed to be equal for the two ion species. The electron multiplier is working in the analogue mode.

\section{Equations governing the ion currents}

In spite of a selective photoexcitation, cascading radiative de-excitation of the directly pumped level $(n l)$ gives an appreciable population of the lower states $\left(n^{\prime} l^{\prime}\right)$. Their densities are correlated to the $n l$ concentration by the following linear system using the Einstein coefficients $A_{i \rightarrow j}$ :

$$
\left[n^{\prime} l^{\prime}\right]=\left(\sum_{p} A_{p \rightarrow n^{\prime} l^{\prime}}[p]\right)\left(\sum_{q} A_{n^{\prime} l^{\prime} \rightarrow q}\right)^{-1} .
$$

The square brackets indicate a density and $p$ (or $q$ ) the levels above (or below) the $\left(n^{\prime} l^{\prime}\right)$ state considered. The Einstein coefficients have been calculated by Gounand (1979) using the Bates-Damgaard theoretical results.

The calculated relative densities obtained for each $(n l)$ level studied are summarised in table 1 . They have been checked experimentally by fluorescence measurements showing that collisional excitation transfer does not play an important role in the pressure range studied. This result is not surprising as the pressure is low and the energy gaps between the different excited states are large.

Table 1. Population densities due to radiative de-excitation.

\begin{tabular}{lcccccccccccc}
\hline Level & $7 \mathrm{~d}$ & $8 \mathrm{p}$ & $5 \mathrm{f}$ & $8 \mathrm{~s}$ & $6 \mathrm{~d}$ & $7 \mathrm{p}$ & $4 \mathrm{f}$ & $7 \mathrm{~s}$ & $5 \mathrm{~d}$ & $6 \mathrm{p}$ & $6 \mathrm{~s}$ & $4 \mathrm{~d}$ \\
\hline$\frac{n^{\prime} l^{\prime}}{n l}\left(\times 10^{2}\right)$ & & & & & 100 & 8.8 & 1.6 & 0.8 & 3.2 & 2.9 & 1.0 & 3.3 \\
& & & & 100 & 0 & 34.2 & 0 & 3.3 & 11.8 & 19.9 & 1.1 & 5.3 \\
& 100 & 5.4 & 1.9 & 0.3 & 1.0 & 1.0 & 0.7 & 0.2 & 2.0 & 3.6 & 0.8 & 3.0 \\
\hline
\end{tabular}

It is obvious that the atomic and molecular ions can be produced by the pumped level and by the radiatively populated states. For a given level $\left(n^{\prime} l^{\prime}\right)$ the corresponding currents are:

$$
\begin{aligned}
& I_{1}^{\prime}\left(n^{\prime} l^{\prime}\right)=e V k_{1}\left(n^{\prime} l^{\prime}\right)[5 \mathrm{p}]\left[n^{\prime} l^{\prime}\right] \quad \text { for Penning ionisation } \\
& I_{2}^{\prime}\left(n^{\prime} l^{\prime}\right)=e V k_{2}\left(n^{\prime} l^{\prime}\right)[5 \mathrm{~s}]\left[n^{\prime} l^{\prime}\right] \quad \text { for associative ionisation } \\
& I_{\nu}^{\prime}\left(n^{\prime} l^{\prime}, \lambda_{i}\right)=e V \frac{P_{i}}{S_{i}} \frac{\lambda_{i}}{h c} \sigma\left(n^{\prime} l^{\prime}, \lambda_{i}\right)\left[n^{\prime} l^{\prime}\right] \quad \text { for photoionisation. }
\end{aligned}
$$

In these expressions, $e, c, h$ are the usual notations for the elementary charge, the speed of light and the Planck constant. $V$ is the interaction volume which is equal to the overlapping volume of the laser beams. $k_{1}\left(n^{\prime} l^{\prime}\right), k_{2}\left(n^{\prime} l^{\prime}\right)$ and $\sigma\left(n^{\prime} l^{\prime}, \lambda_{i}\right)$ are respectively the rate coefficients and the photoionisation cross section at $\lambda_{i}$ for the level $\left(n^{\prime} l^{\prime}\right)$. 
The measured atomic and molecular ion currents $I_{1}^{t}(n l)$ and $I_{2}(n l)$, corresponding to the laser excitation of the $(n l)$ level, are the sum of the partial currents $I_{1}^{\prime}\left(n^{\prime} l^{\prime}\right)$, $I_{2}^{\prime}\left(n^{\prime} l^{\prime}\right)$ and $I_{\nu}^{\prime}\left(n^{\prime} l^{\prime}, \lambda_{i}\right)$ for all the levels and wavelengths. They can be written as

$$
\begin{aligned}
& I_{1}^{t}(n l)=e V\left([5 \mathrm{p}] \sum_{n^{\prime} l^{\prime}} k_{1}\left(n^{\prime} l^{\prime}\right) \frac{\left[n^{\prime} l^{\prime}\right]}{[n l]}+\sum_{n^{\prime} l^{\prime}, \lambda_{i}} \sigma\left(n^{\prime} l^{\prime}, \lambda_{i}\right) \frac{P_{i}}{S_{i}} \frac{\lambda_{i}}{h c} \frac{\left[n^{\prime} l^{\prime}\right]}{[n l]}\right)[n l] \\
& I_{2}(n l)=e V[5 \mathrm{~s}] \sum_{n^{\prime} l^{\prime}} k_{2}\left(n^{\prime} l^{\prime}\right) \frac{\left[n^{\prime} l^{\prime}\right]}{[n l]}[n l] .
\end{aligned}
$$

Introducing effective rate coefficients $K_{i}(n l)$ and effective photoionisation cross sections $Q\left(n l, \lambda_{i}\right)$ :

$$
\begin{aligned}
& K_{i}(n l)=\sum_{n^{\prime} l^{\prime}} k_{i}\left(n^{\prime} l^{\prime}\right) \frac{\left[n^{\prime} l^{\prime}\right]}{[n l]} \\
& Q\left(n l, \lambda_{i}\right)=\sum_{n^{\prime} l^{\prime}} \sigma\left(n^{\prime} l^{\prime}, \lambda_{i}\right) \frac{\left[n^{\prime} l^{\prime}\right]}{[n l]}
\end{aligned}
$$

correlated to the radiative equilibrium obtained in the gas, equations (8) and (9) reduce, respectively, to:

$$
\begin{aligned}
& I_{1}^{t}(n l)=e V\left(K_{1}(n l)[5 \mathrm{p}]+\sum_{\lambda_{i}} \frac{P_{i}}{S_{i}} \frac{\lambda_{i}}{h c} Q\left(n l, \lambda_{i}\right)\right)[n l] \\
& I_{2}(n l)=e V K_{2}(n l)[5 \mathrm{~s}][n l] .
\end{aligned}
$$

\section{Experimental procedure}

$Q\left(n l, \lambda_{i}\right)$ are determined from density ratios presented in table 1 and from calculations of photoionisation cross sections by Aymar et al (1976). These results are summarised in table 2. The determination of $K_{1}(n l)$ and $K_{2}(n l)$ from (12) and (13) only requires density measurements of the $5 \mathrm{p}$ and $n l$ concentrations.

These quantities are deduced from the ion current resulting from the photoionisation induced by the Ar ion laser. For this purpose a part of its beam is sent in the interaction volume on a third diameter of the cell (see figure 1).

In a first step the IR dye laser working alone produces the $5 \mathrm{p}$ state and the $\mathrm{Ar}^{+}$ is tuned at $472.7 \mathrm{~nm}$ in the case of the $5 \mathrm{p}^{2} \mathrm{P}_{1 / 2}$ or at $476.5 \mathrm{~nm}$ for the $5 \mathrm{p}^{2} \mathrm{P}_{3 / 2}$ (this laser beam is characterised by $P_{3}, S_{3}, \lambda_{3}$ and the overlapping volume is $V_{3}=1.2 \times$ $10^{-2} \mathrm{~cm}^{3}$ ). The resulting photoionisation current is

$$
I^{+}=e V_{3} \frac{P_{3}}{S_{3}} \frac{\lambda_{3}}{h c} \sigma\left(5 \mathrm{p}, \lambda_{3}\right)[5 \mathrm{p}]
$$

where $\sigma\left(5 \mathrm{p}, \lambda_{3}\right)$ is known from the experimental results of Moskvin (1963) in good agreement with the theoretical calculations of Aymar et al (1976), [5p] is deduced from $I^{+}$with a good accuracy.

In a second step the $\mathrm{Ar}^{+}$laser $\left(P_{4}, S_{4}, \lambda_{4}\right.$, overlapping volume with the two dye laser beams, $\left.V_{4}=7 \times 10^{-3} \mathrm{~cm}^{3}\right)$ tuned at $\lambda_{4}=514.5 \mathrm{~nm}$, pumps the visible dye and photoionises all the excited levels except the $5 \mathrm{p}$ state. The atomic ion current $I_{1}^{t}$ due to Penning ionisation and to photoionisation by the two dye laser beams is increased by $\Delta I^{+}$. From the effective photoionisation cross section $Q\left(n l, \lambda_{4}\right)$ associated to the 
Table 2. (a) Individual photoionisation cross sections, and, $(b)$ effective photoionisation cross sections, both in units of $10^{-18} \mathrm{~cm}^{2}$.

\begin{tabular}{|c|c|c|c|c|c|}
\hline $\begin{array}{l}\text { (a) } \\
\text { Levels }\end{array}$ & $\sigma(7802 \AA)$ & $\sigma(630 \mathrm{~nm})$ & $\sigma(616 \mathrm{~nm})$ & $\sigma(572 \mathrm{~nm})$ & $\sigma(517 \mathrm{~nm})$ \\
\hline $7 d$ & 5.7 & & & 2.3 & 2.05 \\
\hline $8 p$ & 2.1 & & & 2.0 & 1.7 \\
\hline $5 f$ & 1.0 & & & 0.32 & 0.25 \\
\hline $8 s$ & 0.015 & & $<10^{-3}$ & $<10^{-3}$ & $<10^{-3}$ \\
\hline $6 d$ & 8.0 & 6.4 & 6.0 & 5.0 & 3.3 \\
\hline $7 p$ & 7.0 & 5.5 & 5.0 & 4.0 & 3.15 \\
\hline $4 f$ & 2.0 & 0.7 & 0.55 & 0.45 & 0.35 \\
\hline $7 s$ & 0.1 & 0.03 & 0.015 & $<10^{-2}$ & $<10^{-3}$ \\
\hline $5 \mathrm{~d}$ & 16.0 & 12.0 & 10.1 & 9.8 & 8.0 \\
\hline $6 p$ & 15.0 & 13.0 & 10.8 & 9.5 & 7.5 \\
\hline $6 s$ & 0.0 & 0.25 & 0.20 & 0.18 & 0.15 \\
\hline $4 d$ & 0.0 & 27.0 & 25.0 & 18.0 & 15.0 \\
\hline \multicolumn{6}{|l|}{ (b) } \\
\hline Levels & $Q(n l, 780)$ & $Q\left(n l, \lambda_{2}\right)$ & $Q(n l, 515)$ & & \\
\hline $6 d$ & 9.5 & $\begin{array}{l}7.5 \\
\left(\lambda_{2}=630 \mathrm{~nm}\right)\end{array}$ & 4.8 & & \\
\hline $8 s$ & 6.5 & $\begin{array}{l}6.4 \\
\left(\lambda_{2}=616 \mathrm{~nm}\right)\end{array}$ & 4.7 & & \\
\hline $7 d$ & 6.7 & $\begin{array}{l}3.8 \\
\left(\lambda_{2}=572 \mathrm{~nm}\right)\end{array}$ & 3.3 & & \\
\hline
\end{tabular}

$n l$ state $\Delta I^{+}$can be written:

$$
\Delta I^{+}=e V_{4} \frac{P_{4}}{S_{4}} \frac{\lambda_{4}}{h c} Q\left(n l, \lambda_{4}\right)[n l]
$$

$\Delta I^{+}$is typically a tenth of $I_{1}^{t}$, both are measured simultaneously by chopping (not synchronously) the IR and the $\mathrm{Ar}^{+}$laser beams, the ion current being recorded on a multichannel analyser.

Under these conditions, all the parameters required to determine $K_{1}(n l)$ and $K_{2}(n l)$ from (12) and (13) are known. Nevertheless, these basic formulae assume that the three mechanisms considered are dominant.

The variations of $I_{1}^{\mathrm{t}}$ and $I_{2}$ when plotted against the laser powers $P_{1}$ and $P_{2}$ and against the pressure check this hypothesis. It is shown experimentally that [5p] varies as $P_{1}$ and $[n l]$ varies as $P_{1} P_{2}$ for laser powers up to $320 \mathrm{~mW}$. Consequently, the molecular ion current and the various partial atomic ion currents, i.e. Penning collisional current $I_{1}$, IR photoionisation current $I_{\nu}\left(n l, \lambda_{1}\right)$ and visible photoionisation current $I_{\nu}\left(n l, \lambda_{2}\right)$, vary with $P_{1}$ and $P_{2}$ in the following manner:

$$
\begin{aligned}
& I_{2}(n l)=e V K_{2}(n l)[5 \mathrm{~s}][n l] \sim P_{1} P_{2} \\
& I_{1}(n l)=e V K_{1}(n l)[5 \mathrm{p}][n l] \sim P_{1} \times P_{1} P_{2} \\
& I_{\nu}\left(n l, \lambda_{1}\right)=e V \frac{P_{1}}{S_{1}} \frac{\lambda_{1}}{h c} Q\left(n l, \lambda_{1}\right)[n l] \sim P_{1} \times P_{1} P_{2} \\
& I_{\nu}\left(n l, \lambda_{2}\right)=e V \frac{P_{2}}{S_{2}} \frac{\lambda_{2}}{h c} Q\left(n l, \lambda_{2}\right)[n l] \sim P_{2} \times P_{1} P_{2} .
\end{aligned}
$$


Therefore, if one of the powers is kept constant, for instance $P_{2}$, the variation of

$$
I_{1}^{\dagger}(n l)=I_{1}(n l)+I_{\nu}\left(n l, \lambda_{1}\right)+I_{\nu}\left(n l, \lambda_{2}\right)
$$

against $P_{1}$ should be the sum of a linear and a quadratic term. The linear part $L_{1}$ comes from the photoionisation by the visible radiation, the quadratic term $Q_{1}$ comes from the Penning ionisation and from the photoionisation induced by the IR beam. At constant $P_{1}$, the Penning ionisation and the IR photoionisation provide a linear term $L_{2}$, the visible photoionisation a quadratic term $Q_{2}$ : when the constant power is changed the linear and quadratic terms exchange their mechanisms. For a given set of powers $P_{1}$ and $P_{2} I_{1}^{t}(n l)$ is

or

$$
I_{1}^{t}(n l)=P_{1}+Q_{1}=l_{1}\left(P_{2}\right) P_{1}+q_{1}\left(P_{2}\right) P_{1}^{2}
$$

$$
I_{1}^{t}(n l)=P_{2}+Q_{2}=l_{2}\left(P_{1}\right) P_{2}+q_{2}\left(P_{1}\right) P_{2}^{2}
$$

where $l_{i}$ and $q_{i}$ are parameters to be determined.

Experimental investigation of these dependences confirms the validity of the mechanisms considered and rules out other reactions which have not the proper variation with the two laser powers. These possible processes are discussed further.

Experimentally, at different pressures, the laser powers are varied using density filters fixed on a rotating wheel and the ion currents are recorded synchronously. This technique allows us to determine the $l_{i}$ and $q_{i}$ values from a least-squares fit method with a good correlation factor. The ratios between the linear and the quadratic parts of the atomic ion current for given laser powers can be written

$$
\frac{Q_{1}}{L_{1}}=\frac{L_{2}}{Q_{2}}=\frac{P_{1}}{P_{2}} \frac{S_{2}}{S_{1}} \frac{\lambda_{1}}{\lambda_{2}} \frac{Q\left(n l, \lambda_{1}\right)}{Q\left(n l, \lambda_{2}\right)}+K_{1}(n l)[5 \mathrm{p}]\left(\frac{P_{2}}{S_{2}} \frac{Q\left(n l, \lambda_{2}\right) \lambda_{2}}{h c}\right)^{-1} \text {. }
$$

At a given pressure, the shape of the atomic ion current curve and the linear and quadratic term exchange show that the only possible mechanisms are (1), (2) and (3). The experimental results give on one side the photoionisation current induced by the visible laser and on the other side the IR photoionisation current plus the Penning ionisation current. The relative importance of these two last currents is determined from the theoretical photoionisation cross sections. The study of the pressure dependence confirms the validity of the cross sections selected: at a given laser power $I_{\nu}\left(n l, \lambda_{1}\right)$ is proportional to $n l$, and $I_{1}(n l)$ to $[n l][5 \mathrm{p}]$, so when the pressure increases and consequently [5p], $I_{1}(n l)$ increases as the square of $I\left(n l, \lambda_{1}\right)$. This last study demonstrates the validity of the hypothesis.

\section{Determination of the rate coefficients}

Measurements of the atomic and molecular ion currents as a function of the laser powers and of the pressure allow us to determine $K_{1}(n l)$ and $K_{2}(n l)$ unambiguously from (12) and (13). The experimental procedure gives the rate coefficients by different methods.

(a) From (12) and (15) the following form of $K_{1}(n l)$ can be deduced

$$
K_{1}(n l)=\left(\frac{I_{1}^{\mathrm{t}}}{\Delta I^{+}}-\alpha_{1} \frac{P_{1}}{P_{4}}-\alpha_{2} \frac{P_{2}}{P_{4}}\right) \frac{\beta P_{4}}{[5 \mathrm{p}]}
$$


with

and

$$
\alpha_{i}=\frac{V}{V_{4}} \frac{\lambda_{i}}{\lambda_{4}} \frac{Q\left(n l, \lambda_{i}\right)}{Q\left(n l, \lambda_{4}\right)} \frac{S_{4}}{S_{i}}
$$

$$
\beta=\frac{V_{4}}{V} \frac{\lambda_{4}}{h c S_{4}} Q\left(n l, \lambda_{4}\right)
$$

Here, the main experimental quantity is $I_{1} / \Delta I^{+}$. It is obtained by a chopping technique and a simultaneous measurement of the currents. This experimental procedure correlates the photoionisation currents $I_{\nu}\left(n l, \lambda_{i}\right)$ induced by the infrared and the visible laser beams to the photoionisation current $\Delta I^{+}$given by the argon ion laser tuned at $514.5 \mathrm{~nm}$ :

$$
I_{\nu}\left(n l, \lambda_{i}\right)=\alpha_{i} \Delta I^{+} P_{i} / P_{4} .
$$

It is an experimental check of the importance of the photoionisation mechanisms.

(b) $K_{1}(n l)$ can be also determined from (18):

$$
K_{1}(n l)=\left(\frac{Q_{1}}{L_{1}}-\gamma \frac{P_{1}}{P_{2}}\right) \frac{\delta P_{2}}{[5 \mathrm{p}]}
$$

with

$$
\gamma=\frac{\lambda_{1}}{\lambda_{2}} \frac{Q\left(n l, \lambda_{1}\right)}{Q\left(n l, \lambda_{2}\right)} \frac{S_{2}}{S_{1}} \quad \text { and } \quad \delta=\frac{\lambda_{2}}{h c S_{2}} Q\left(n l, \lambda_{2}\right)
$$

Here, the main experimental quantity is $Q_{1} / L_{1}$ (or $L_{2} / Q_{2}$ ). This is determined by a modulation technique and a least squares fit. The elementary processes and the effective rate coefficient are obtained simultaneously. It should be noticed that the interaction volume between the IR and the visible laser beams does not appear in formula (20).

The accuracy of the two methods is comparable. Their results are in good agreement and complementary. The experimental results presented in this paper for $K_{1}(n l)$ are the average values obtained by these two approaches.

(c) The associative rate coefficient $K_{2}(n l)$ can be written from (13) and (15) as

$$
K_{2}(n l)=\frac{I_{2}}{\Delta I^{+}} P_{4} \frac{\beta}{[5 \mathrm{p}]}
$$

The linear dependence of $\mathrm{Rb}_{2}^{+}$on the laser powers is checked by the modulation technique.

\section{Power measurements}

The determination of $K_{1}(n l)$ and $K_{2}(n l)$ needs the knowledge of the laser powers $P_{i}$ at different wavelengths $\lambda_{i}$ in the interaction zone. $\lambda_{2}, \lambda_{3}$ and $\lambda_{4}$ do not correspond to transitions to the ground state and consequently are slightly absorbed in the gas. The main absorption is on the entrance and the exit window of the cell: the measurement of the input $E_{i}$ and output $O_{i}$ powers allows the calculation of the laser power $P_{i}$ :

$$
P_{i}=\left(1-A_{i}\right) E_{i}=\left(E_{i} O_{i}\right)^{1 / 2}
$$

assuming the same absorption $\boldsymbol{A}_{i}$ for each window. 
For the resonance line $\lambda_{1}$ the measurement is more difficult. A strong absorption occurs at $\lambda_{1}$ in the first few millimeters into the cell (the interaction area is located $12.5 \mathrm{~cm}$ away from the entrance window). However, the photoionisation is produced by the whole laser line which is wider $(25 \mathrm{GHz})$ than the absorption line (Doppler width $1 \mathrm{GHz}$ ). Consequently, the influence of the resonant absorption is not very important. The laser power, in the interaction zone, is determined by taking into account the absorption due to the window and correcting the entrance power from the resonance absorption $A_{\mathrm{R}}$. For this purpose, entrance and exit powers are measured at the resonance $\left(E_{1}, O_{1}\right)$ and out of resonance $\left(E^{\prime}, O^{\prime}\right) . E_{1}, O_{1}, E^{\prime}$ and $O^{\prime}$ are related by:

$$
O_{1}=\left(1-A_{\mathrm{R}}\right)\left(1-A_{1}\right)^{2} E_{1} \quad O^{\prime}=\left(1-A_{1}\right)^{2} E^{\prime}
$$

and give

$$
P_{1}=\left(1-A_{\mathrm{R}}\right)\left(1-A_{1}\right) E_{1}=O_{1}\left(E^{\prime} / O^{\prime}\right)^{1 / 2}
$$

\section{Experimental results}

The $6 d^{2} D_{3 / 2}, 6 d^{2} D_{5 / 2}, 8 s^{2} S_{1 / 2}$ and $7 d^{2} D_{5 / 2}$ levels have been excited from the $5 p^{2} P_{3 / 2}$ level, and $6 \mathrm{~d}^{2} \mathrm{D}_{3 / 2}$ from $5 \mathrm{p}^{2} \mathrm{P}_{1 / 2}$. The $7 \mathrm{~d}^{2} \mathrm{D}_{3 / 2}$ has an absorption coefficient which is too low and is energetically too close to the $7 \mathrm{~d}^{2} \mathrm{D}_{5 / 2}$ level to be excited alone. The $\mathrm{d}$ and s levels are pumped using $\mathrm{Rh} 6 \mathrm{G}$, the $5 \mathrm{p}$ using oxazine 2 .

Density measurements and rate coefficient determinations have been performed in the pressure range $10^{-5}$ to $10^{-3}$ Torr. Figure 2 represents $5 \mathrm{p}$ and $8 \mathrm{~s}$ concentrations plotted against pressure at constant laser powers. It should be noticed that [8s] varies as $[5 p]$ and that the shape of the [5p] curve differs from a linear dependence. This

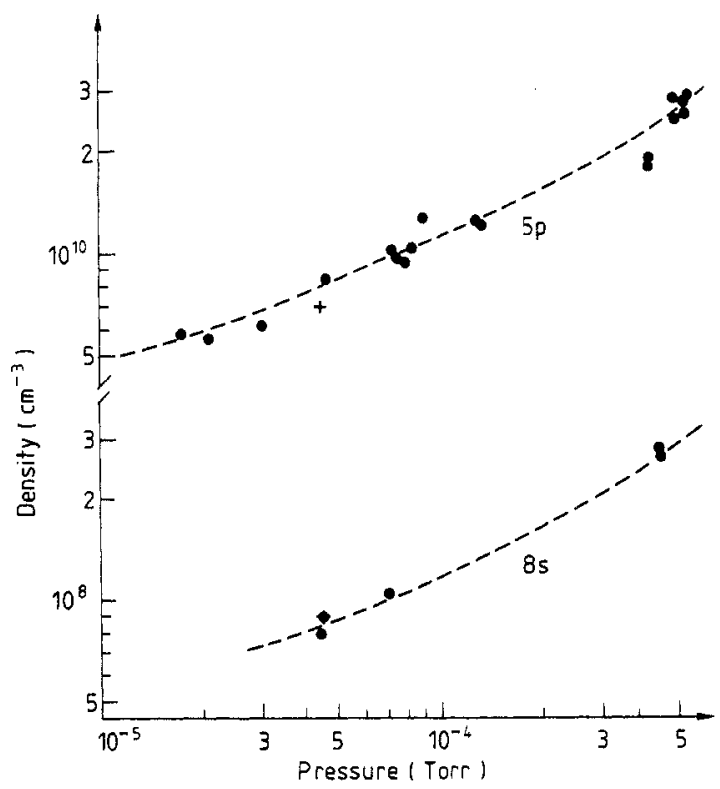

Figure 2. $5 \mathrm{p}^{2} \mathrm{P}_{3 / 2}$ and $8 \mathrm{~s}^{2} \mathrm{~S}_{1 / 2}$ concentrations plotted against pressure at $P_{1}=164 \mathrm{~mW}$ and $P_{2}=216 \mathrm{~mW}$. 
can be explained by the strong radiation trapping of the resonance line. Density measurements avoid the difficult theoretical treatment of excitation by a resonance line.

Density measurements have been performed at different powers and different pressures to check the dependences upon $P_{1}$ and $P_{2}$. As the density ratios $[5 \mathrm{p}] /[5 \mathrm{~s}]$ (calculated from figure 2 ) and $[n l] /[5 \mathrm{p}]$ are low, i.e. far from saturation values, it is not surprising that [5p] varies as $P_{1}$ and $[n l]$ as $P_{1} P_{2}$. The following values have been obtained for the $[n l] /[5 \mathrm{p}]$ ratios: $1: 390$ for $6 \mathrm{~d}^{2} \mathrm{D}_{3 / 2}$ at $P_{2}=120 \mathrm{~mW}, 1: 54$ for $6 \mathrm{~d}^{2} \mathrm{D}_{5 / 2}$ $(120 \mathrm{~mW}), 1: 78$ for $8 \mathrm{~s}^{2} \mathrm{~S}_{1 / 2}(216 \mathrm{~mW}), 1: 53$ for $7 \mathrm{~d}^{2} \mathrm{D}_{5 / 2}(108 \mathrm{~mW})$ pumped from the $5 \mathrm{p}^{2} \mathrm{P}_{3 / 2}$ level, and $1: 48$ for the $6 \mathrm{~d}^{2} \mathrm{D}_{3 / 2}$ pumped from the $5 \mathrm{p}^{2} \mathrm{P}_{1 / 2}\left(P_{2}=152 \mathrm{~mW}\right)$.

The experimental variations of $I_{1}$ and $I_{2}$ plotted against $P_{1}$ and $P_{2}$ are consistent with the prediction of formulae (16). Figure 3 represents a typical variation obtained for the $7 \mathrm{~d}^{2} \mathrm{D}_{5 / 2}$ level at intermediate pressure, $p=4 \times 10^{-4}$ Torr. The behaviour of the $\mathrm{Rb}^{+}$curves corresponds to the sum of a linear and a quadratic term which exchange when the constant power is changed. The whole procedure can be applied to determine $K_{1}(n l)$ and $K_{2}(n l)$.

The effective rate coefficients as calculated from (19) or (20) and from (21) are summarised in table 3 . The differences between $K_{1}(n l)$ calculated from (19) or (20)

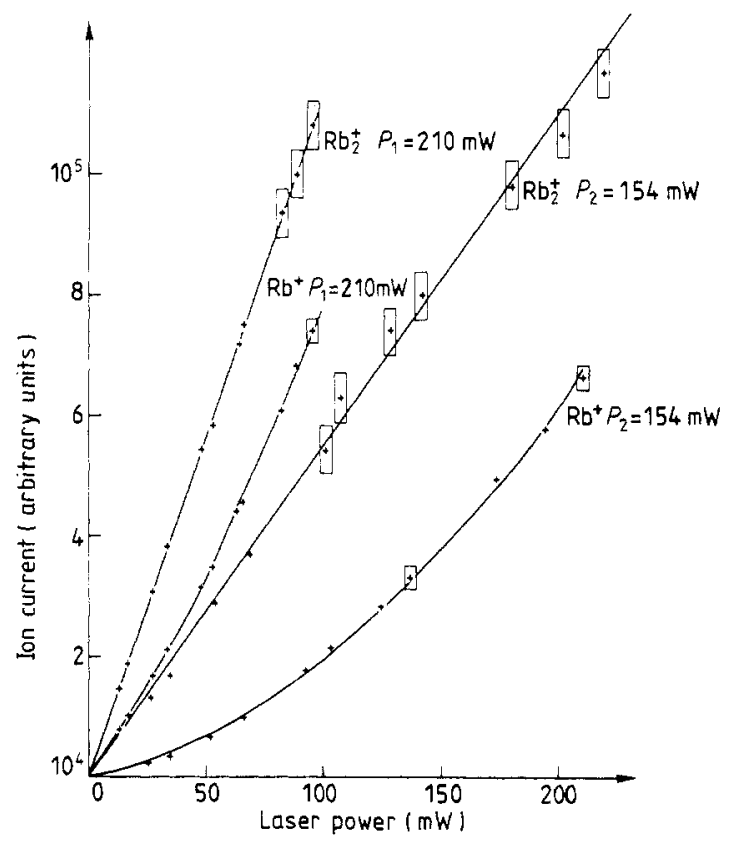

Figure 3. Ion currents plotted against $P_{1}$ and $P_{2}$ for the $7 \mathrm{~d}^{2} \mathrm{D}_{5 / 2}$ at $4.7 \times 10^{-5}$ Torr.

Table 3. Effective collisional rate coefficients $K_{i}(n l)$ and individual rate coefficients $k_{i}(n l)$.

\begin{tabular}{lllll}
\hline Level & $\begin{array}{l}K_{1}(n l) \\
\left(10^{-8} \mathrm{~cm}^{3} \mathrm{~s}^{-1}\right)\end{array}$ & $\begin{array}{l}K_{2}(n l) \\
\left(10^{-10} \mathrm{~cm}^{3} \mathrm{~s}^{-1}\right)\end{array}$ & $\begin{array}{l}k_{\mathrm{t}}(n l) \\
\left(10^{-8} \mathrm{~cm}^{3} \mathrm{~s}^{-1}\right)\end{array}$ & $\begin{array}{l}k_{2}(n l) \\
\left(10^{-10} \mathrm{~cm}^{3} \mathrm{~s}^{-1}\right)\end{array}$ \\
\hline $6 \mathrm{~d}^{2} \mathrm{D}_{3 / 2}$ & $4.9 \pm 1.0$ & $2.0 \pm 0.5$ & $4.2 \pm 0.9$ & $2.0 \pm 0.5$ \\
$6 \mathrm{~d}^{2} \mathrm{D}_{5 / 2}$ & $4.8 \pm 1.0$ & $1.7 \pm 0.4$ & $4.1 \pm 0.9$ & $1.7 \pm 0.4$ \\
$8 \mathrm{~s}^{2} \mathrm{~S}_{1 / 2}$ & $3.7 \pm 1.2$ & $2.5 \pm 0.6$ & $2.2 \pm 0.7$ & $2.5 \pm 0.6$ \\
$7 \mathrm{~d}^{2} \mathrm{D}_{5 / 2}$ & $2.6 \pm 1.0$ & $4.4 \pm 0.9$ & $2.2 \pm 0.9$ & $4.2 \pm 0.9$ \\
\hline
\end{tabular}


are not significant. This strongly supports the calculated values of the photoionisation cross sections. No variation of the rate coefficients with the pressure has been observed. Figure 4 gives for the $8 \mathrm{~s}$ level the collisional atomic current $I_{1}(8 \mathrm{~s})$, the molecular current $I_{2}(8 \mathrm{~s})$ and the total collisional current $I_{1}(8 \mathrm{~s})+I_{2}(8 \mathrm{~s})$ plotted against pressure. Full points and crosses are deduced from measurements, the full and broken curves are calculated with the same values of $K_{1}(n l)$ and $K_{2}(n l)$ at different pressures with the densities deduced from figure 2 . Changing $K_{i}(n l)$ induces a parallel shift of the curves. The same rate coefficients explain all the measurements.

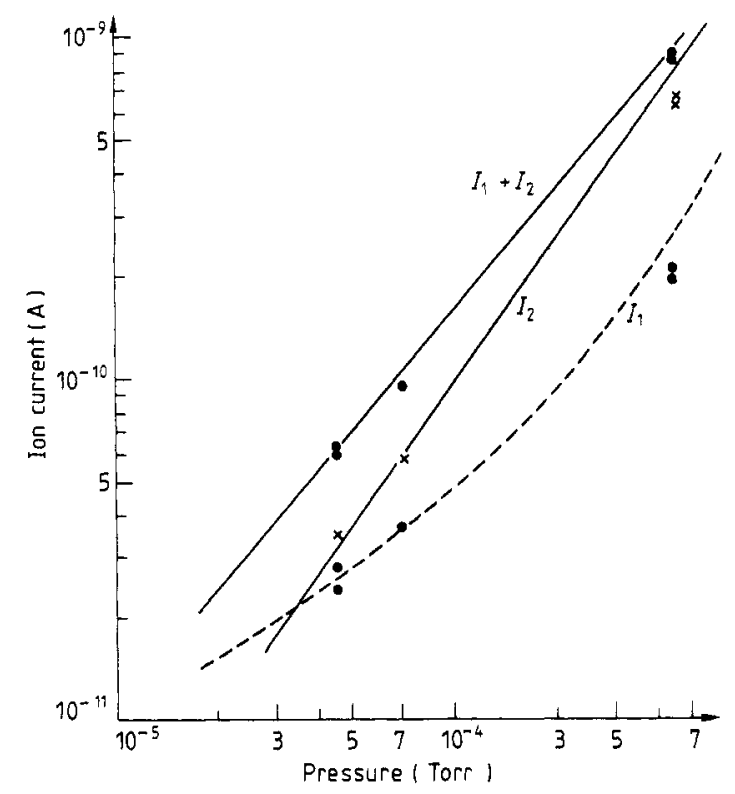

Figure 4. Ion collisional currents plotted against pressure for the $8 \mathrm{~s}^{2} \mathrm{~S}_{1 / 2}$ level at $P_{1}=$ $154 \mathrm{~mW}$ and $P_{2}=210 \mathrm{~mW}$.

\section{Individual rate coefficients}

It is easy to deduce the individual associative rate coefficients $k_{2}(n l)$ for the pumped level from the effective coefficients $K_{2}(n l)$.

In figure 5 the $\mathrm{Rb}_{2}^{+}$limit corresponding to a potential well of $5300 \mathrm{~cm}^{-1}$ for this ion is reported, as calculated by Valance (1978). The $6 \mathrm{~d}$ levels are the lowest for which associative ionisation is energetically possible: consequently $K_{2}(6 \mathrm{~d})$ is equal to $k_{2}(6 \mathrm{~d})$. As the $8 \mathrm{~s} \rightarrow 6 \mathrm{~d}$ transition is optically forbidden and as the collisional transfers are negligible there is no level below $8 \mathrm{~s}$ which contributes to associative ionisation: $K_{2}(8 \mathrm{~s})$ is also equal to $k_{2}(8 \mathrm{~s})$.

When the $7 \mathrm{~d}$ level is excited the $8 \mathrm{p}, 5 \mathrm{f}, 8 \mathrm{~s}$ and $6 \mathrm{~d}$ levels also contribute to the molecular ion current. $k_{2}(6 \mathrm{~d})$ and $k_{2}(8 \mathrm{~s})$ have been determined. Klucharev et al (1980) gives an experiental rate coefficient for the $8 \mathrm{p}$ level close to the value presented here for the $6 \mathrm{~d}$ and $8 \mathrm{~s}$ levels. These different measurements indicate that there is no effect of the orbital quantum number for highly excited atoms. This last conclusion has been extended to the $5 f$ level. An interpolation against the effective quantum 


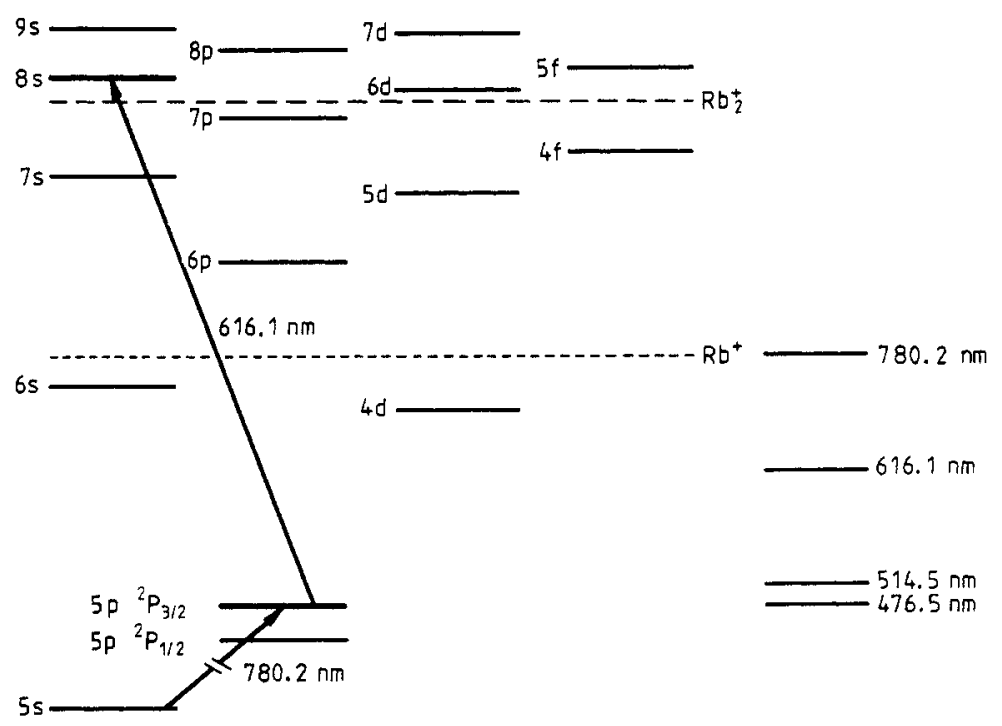

Figure 5. Energy diagram of the Rb levels showing a two-step excitation of the $8 \mathrm{~s}$ level. The upper broken line represents the lowest energy limit for associative ionisation and the lower broken line the limit for Penning ionisation with a $5 p^{2} \mathrm{P}_{3 / 2}$ level. The full lines on the right-hand side represent the lowest photoionisation energy limit at the corresponding wavelengths.

number $n^{*}$ between $k_{2}(8 \mathrm{~s})$ and $k_{2}(8 p)$ has been done to determine $k_{2}(5 \mathrm{f})$ (figure 6). In fact the $5 \mathrm{f}$ density is quite low and the correction is weak. The $k_{2}(n l)$ values are summarised in table 3 .

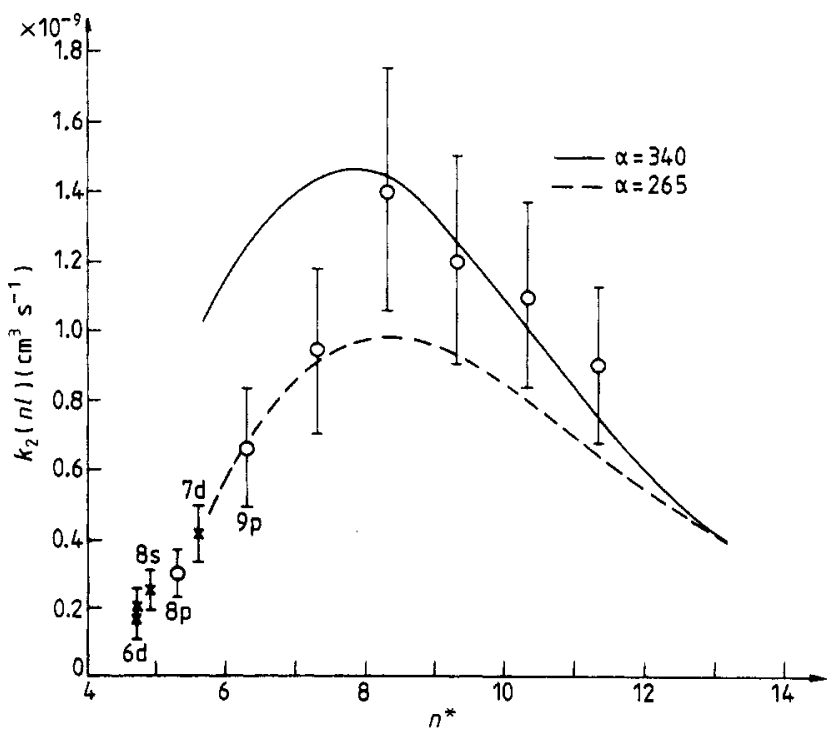

Figure 6. The $k_{2}(n l)$ variation plotted against $n^{*}$. The full and broken curves are the Mihajlov and Janev (1981) theoretical curves for two values of the Rb polarisability $\alpha$ : ,$- \alpha=340 ;---, \alpha=265$. The experimental data are; $\bigcirc$, Klucharev et al $(1980) ; \times$, this work. 
All the levels above the 6s level can contribute to the Penning ion current and no rate coefficient is available except the present $K_{1}(n l)$ values. Consequently, $k_{1}(n l)$ values are more difficult to obtain. Below the directly pumped level the most populated are the $\mathrm{p}$ states (table 1). They contribute strongly to the population equilibrium when the $8 \mathrm{~s}$ level is pumped and more slightly in the case of a d level. As there is no significant difference between $K_{1}(8 \mathrm{~s}), K_{1}(6 \mathrm{~d})$ or $K_{1}(7 d), k_{1}(n p)$ is certainly not much greater than $k_{1}(n \mathrm{~d})$. The same remark can be done for the $4 \mathrm{f}$ level taking into account $K_{1}(6 \mathrm{~d}), K_{1}(7 \mathrm{~d})$ and the relative concentrations. As no level seems to play a dominant role $K_{1}(6 \mathrm{~d}), K_{1}(8 \mathrm{~s})$ and $K_{1}(7 \mathrm{~d})$ are corrected from the population distribution (table 1$)$ to determine $k_{1}(6 \mathrm{~d}), k_{1}(8 \mathrm{~s})$ and $k_{1}(7 \mathrm{~d})$. These values are reported in table 3 .

The neighbouring levels of the $6 \mathrm{~d}$ or $7 \mathrm{~d}$ states have low relative concentrations and as a matter of fact $k_{1}(n \mathrm{~d})$ differs slightly from $K_{1}(n \mathrm{~d})$. On the other hand, the neighbouring levels are very important for the $8 \mathrm{~s}$ level. If $k_{1}(8 \mathrm{~s})$ and $k_{1}(7 \mathrm{~s})$ are assumed negligible a common value of $5.6 \times 10^{-8} \mathrm{~cm}^{3} \mathrm{~s}^{-1}$ is obtained for $k_{1}(7 \mathrm{p}), k_{1}(5 \mathrm{~d})$ and $k_{1}(6 \mathrm{p})$, whereas $k_{1}(6 \mathrm{~d})$ and $k_{1}(7 \mathrm{~d})$ are weakly modified. As this large value is not inconsistent with the $6 \mathrm{~d}$ and $7 \mathrm{~d}$ measurements, an unambiguous determination of $k_{1}(n \mathrm{~s})$ needs complementary measurements.

\section{Accuracy of the method}

Error bars corresponding to measurement dispersion are indicated in table 3 . Different parameters are used to determine $K_{1}$ and $K_{2}$. Some of these, the photoionisation cross sections and the Einstein coefficient are calculated from theoretical works. Only the ratios of theoretical data are used except for the $5 \mathrm{p}$ photoionisation cross section which is also known from measurements. The fluorescence intensity measurements and the photoionisation currents confirm the Einstein coefficient ratios and the variation of the photoionisation cross section with wavelength. The rate coefficients can be considered as reliable from that point of view.

Other parameters such as interaction volumes and laser powers are correlated to experimental diagnostics. The determination of [5p] requires knowledge of the interaction volume between the IR and the $\mathrm{Ar}^{+}$laser beams. This volume has been checked by moving the $\mathrm{Ar}^{+}$laser beam transversally through the IR beam. The $5 \mathrm{p}$ distribution has been found to coincide with the IR beam diameter. Only interaction volume ratios are used in formulae (19) and (21). Volumes do not appear in formula (20). Under these conditions the apparatus can be considered as giving absolute values of the rate coefficients.

\section{Neglected mechanisms}

The experimental variations of $I_{1}$ and $I_{2}$ with $P_{1}$ and $P_{2}$ show that various mechanisms of ion production are negligible under our experimental conditions.

(A) The linear shape of $\mathrm{Rb}_{2}^{+}$rules out the following (and similar) mechanisms with a quadratic behaviour with the powers.

(a) associative ionisation between excited states:

$$
\begin{array}{ll}
\mathrm{Rb}(5 \mathrm{p})+\mathrm{Rb}(n l) \rightarrow \mathrm{Rb}_{2}^{+}+\mathrm{e}^{-} & \text {rate coefficient } k_{4} \\
\mathrm{Rb}(n l)+\mathrm{Rb}\left(n^{\prime} l^{\prime}\right) \rightarrow \mathrm{Rb}_{2}^{+}+\mathrm{e}^{-} & \text {rate coefficient } k_{5} .
\end{array}
$$


An upper limit of the reaction rates can be estimated by assuming the linearity to be better than $10 \%, k_{4}$ should be less than $2 \times 10^{-9} \mathrm{~cm}^{3} \mathrm{~s}^{-1}$ and $k_{5}$ than $2 \times$ $10^{-7} \mathrm{~cm}^{3} \mathrm{~s}^{-1}$. This last value is too large to have a physical meaning but the upper limit obtained for $k_{4}$ shows that associative ionisation between two excited states is less than a tenth of the Penning ionisation. Such a result has often been obtained in Penning ionisation studies (Manus et al 1977, Pesnelle et al 1981).

(b) Photodissociation of $\mathrm{Rb}_{2}^{+}$:

$$
\mathrm{Rb}_{2}^{+}+h \nu \rightarrow \mathrm{Rb}^{+}+\mathrm{Rb} .
$$

An upper limit of $5 \times 10^{-17} \mathrm{~cm}^{2}$ can be determined for the cross section of this mechanism. This is not at variance with the experimental results of Bearman and Leventhal (1978) who estimate the photodissociation cross section of $\mathrm{Na}_{2}^{+}$to be $10^{-17} \mathrm{~cm}^{2}$.

The lack if photodissociation has also been checked with the $\mathrm{Ar}^{+}$laser beam.

(B) The variation of the atomic ion current with the visible and the IR powers allows us to discard the following (and similar) mechanisms.

(a) Penning ionisation between two highly excited states:

$$
\mathrm{Rb}(n l)+\mathrm{Rb}\left(n^{\prime} l^{\prime}\right) \rightarrow \mathrm{Rb}^{+}+\mathrm{e}^{-}+\mathrm{Rb}
$$

which varies quadratically with the two laser powers.

The upper limit of the rate coefficient is estimated to be $10^{-7} \mathrm{~cm}^{3} \mathrm{~s}^{-1}$.

(b) Ion pair formation:

$$
\mathrm{Rb}(n l)+\mathrm{Rb}(5 \mathrm{~s}) \rightarrow \mathrm{Rb}^{+}+\mathrm{Rb}^{-}
$$

which varies linearly with the two laser powers.

From the electron affinity of the negative rubidium ion (Frey et al 1978) this reaction is not energetically possible for the $6 \mathrm{~d}$ and $8 \mathrm{~s}$ levels. The rate coefficient for the $7 \mathrm{~d}$ level is estimated to be less than $8 \times 10^{-12} \mathrm{~cm}^{3} \mathrm{~s}^{-1}$.

Other mechanisms can be ruled out by calculations or experimental observations.

(C) Ionisation by hot electrons:

$$
\mathrm{Rb}(n l)+\mathrm{e}(0.9<\varepsilon<1.75 \mathrm{eV}) \rightarrow \mathrm{Rb}^{+}+\mathrm{e} .
$$

With the laser powers used, these electrons are mainly formed by photoionisation . A calculation, for the 8s level, using the Percival theory (1966) gives an electron impact ionisation cross section of $3 \times 10^{-14} \mathrm{~cm}^{2}$ at an electron energy of $1.5 \mathrm{eV}$. Such a cross section provides ion currents three orders of magnitude less than the total measured atomic current.

(D) The ion currents collected with only the IR laser beam tuned at the resonance are also at least three orders of magnitude less than the measured currents. No current is detected when this laser is detuned.

\section{Discussion and conclusion}

The experimental associative rate coefficients obtained for $\mathrm{s}$ or $\mathrm{d}$ levels are of the same order of magnitude as the coefficients measured by Klucharev (1980) for $p$ levels. This seems to indicate a negligible influence of the orbital quantum number on the cross section value. Figure 6 gives the set of experimental results together 
with the theoretical curves obtained by Mihajlov and Janev (1981) for two values of the $\mathrm{Rb}$ polarisability.

However, it should be noticed that the calculated curves are only valid for $p$ and d levels. The Mihajlov model gives results depending on the photoionisation cross section near the threshold. The Kramer approximation used by Mihajlov is reasonable for the calculation of the $\mathrm{p}$ or $\mathrm{d}$ photoionisation cross sections near the threshold but gives results about ten times too high for the s levels. Actually, the theoretical value of $k_{2}(n \mathrm{~s})$ should rather be ten times lower than $k_{2}(n \mathrm{p})$ or $k_{2}(n \mathrm{~d})$, in disagreement with the experimental observation.

Lower values of associative ionisation cross sections for s states have been obtained theoretically and experimentally in helium (Cohen 1976): $k(3 \mathrm{~s}) / k(3 \mathrm{p})=1: 30$ and $k(3 \mathrm{~s}) / k(3 \mathrm{~d})=1: 50$ but also $k(4 \mathrm{~s}) / k(4 \mathrm{~d})=1: 3$ and $k(4 \mathrm{~s}) / k(4 \mathrm{~d})=1: 7$. These data might indicate that when $n$ increases associative ionisation cross sections for an state and a $\mathrm{p}$ or $\mathrm{d}$ state come close together.

Recently Kushawaha and Leventhal (1982) determined rate coefficients of $7.8 \times$ $10^{-11}$ and $3.3 \times 10^{-13} \mathrm{~cm}^{3} \mathrm{~s}^{-1}$ for the associative ionisation of the $4 \mathrm{~d}$ and $5 \mathrm{~s}$ sodium atoms colliding with a ground-state $\mathrm{Na}$ atom (3s). The first coefficient lies in the range of the present measurements and the second seems to agree with the preceding theoretical remarks. The $5 \mathrm{~s}$ and $4 \mathrm{~d}$ levels are the lowest states of $\mathrm{Na}$ for which the associative ionisation with a ground-state atom is energetically possible which prevents any correction for cascading de-excitation. However, this mechanism gives a concentration for the $4 \mathrm{p}$ level which is half that of the $5 \mathrm{~s}$ state. Under these conditions it is highly surprising that the authors do not see any $\mathrm{Na}^{+}$ions. The photoionisation of the $4 p$ level should give an atomic ion current of the same order of magnitude as the measured molecular ion current according to the published 5 s density and laser powers. They also considered the Penning ionisation coefficient to be very low: this is in disagreement with the results of Le Gouët et al (1982). A Penning rate coefficient of the order of $10^{-8} \mathrm{~cm}^{3} \mathrm{~s}^{-1}$ is used by these authors to account for electron energy distribution in excited $\mathrm{Na}$ vapour.

The present experimental work indicates clearly the importance of Penning ionisation when two excited short-lifetime $\mathrm{Rb}$ atoms collide. It would be of a great interest to have a specific theoretical interpretation for (i) Penning ionisation including two excited states, and (ii) associative ionisation especially involving $n$ s states.

\section{Acknowledgments}

The authors are indebted to Dr M Aymar for specific calculations of photoionisation cross sections. They would like to thank Dr J Berlande for helpful discussions and valuable comments. It is a pleasure to thank $R$ Durand for his technical assistance.

\section{References}

Allegrini M, Bicchi P, Gozzini S and Savino P 1981 Opt. Comm. 36449

Aymar M, Luc-Koenig E and Combet-Farnoux F 1976 J. Phys. B: At. Mol. Phys. 91279

Bearman G H and Leventhal J J 1978 Phys. Rev. Lett. 411227

Chéret M, Barbier L, Lindinger W and Deloche R 1982 Chem. Phys. Lett. 88229

Chéret M, Spielfiedel A, Durand R and Deloche R 1981 J. Phys. B: At. Mol. Phys. 143953 
Cohen J S 1976 Phys. Rev. A 1399

Frey P, Breyer F and Hotop H 1978 J. Phys. B: At. Mol. Phys. 11 L589

Gounand F 1979 J. Physique 40457 (Data available on request)

Klucharev A N, Lazarenko A V and Vujvonic V 1980 J. Phys. B: At. Mol. Phys. 131143

Kushawaha V S and Leventhal J J 1982 Phys. Rev. A 25570

Le Gouët J L, Picque J L, Wuillemier F, Bizau J M, Dhez P, Koch P and Ederer D L 1982 Phys. Rev. Lett. 48600

Lucatorto T B and McIlrath T J 1976 Phys. Rev. Lett. 37428

Manus C, Pesnelle A and Watel G 1977 Proc. 10th Int. Conf. on Physics of Electronic and Atomic Collisions (Paris: Commissariat à l'Energie Atomique). Invited Paper p 525

Mihajlov A A and Janev R K $1981 J$. Phys. B: At. Mol. Phys. 141639

Moskvin Yu V 1963 Opt. Spectrosc. 15316

Percival I C 1966 Nucl. Fusion 6182

Pesnelle A, Runge S, Sevin D, Wolffer N and Watel G 1981 J. Phys. B: At. Mol. Phys. 141827

Roussel F, Breger P, Spiess G, Manus C and Geltman S 1980 J. Phys. B: At. Mol. Phys. 13 L631

Roussel F, Carre B, Breger P and Spiess G 1981 J. Phys. B: At. Mol. Phys. 14 L313

Valence A 1978 J. Chem. Phys. 69355 